in the Swedish workforce. Future research should continue to assess sex-stratified relationships, using detailed shift work exposure categories and objective registry data where possible.

\section{SHIFTWORK AND CANCER}

Eugene Waclawski. Occupational Health, University of Edinburgh, UK

\subsection{6/oemed-2018-ICOHabstracts. 1374}

Aim of special session What practical advice can Occupational health practitioners give to those at risk and to management for employees:

a. with no obvious risk factors for cancer other than shiftwork,

b. for those with risk factors (strong FHx, gene markers of susceptibility, obesity, benign breast disease, HRT, late menopause, early menarche, late first birth... for breast cancer),

c. those with cancers under treatment or in remission in relation to nightwork,

Also is there a threshold effect? (years of exposure, number of shifts, hours of nightwork),

Is there any need for concern or preventive measures?:

- Should shift lengths be shorter?;

- Should rest breaks should be included; and

- how should researchers educate shift workers and employers as to how sleep-wake cycles are controlled and

- How can we maximise sleep quality, sleep duration, and alertness at work?

Speakers Prof Eva Schernhammer, Associate Professor, Harvard School of Public Health, Boston, USA,

Professor Johnni Hansen Danish Cancer Registry; johnni@cancer.dk.

Prof Lin Fritschi, Curtain University Australia/lin.fritschi@curtin.edu.au.

Prof Damien McElvenny IOM UK; Damien.McElvenny@iom-world.org.

Prof Shelly Tse Lap Ah, Associate Professor, Centre for Occupational and Environmental Health Studies (COEHS), The Chinese University of Hong Kong/shelly@cuhk.edu.hk

\section{6a CAN WE REDUCE HARM FROM SHIFTWORK?}

Lin Fritschi. Curtin University, Perth, Australia

\subsection{6/oemed-2018-ICOHabstracts. 1375}

The association between shiftwork and cancer has been the focus of a number of case-control and cohort studies since the 2010 declaration by the International Agency for Research on Cancer that shiftwork which involves circadian disruption probably caused breast cancer. There is still debate about the association. However shiftwork has also been associated with a range of other health conditions including fatigue, injury, diabetes and cardiovascular disease. In addition, there has been a lot of interest in whether people with different chronotypes (e.g. morning people versus evening people) react to shiftwork differently. Shiftwork cannot be avoided in our 24 hour society, but we have a responsibility to minimise the health impacts upon shiftworkers. We need to consider what aspects of shiftwork might be related to the different health conditions in order to minimise any effect on workers. Thus, whether shiftwork causes breast cancer or not, there is still useful advice we can give shiftworkers in order to improve the quality of life of those working night shifts. This talk will review some of the potential interventions to improve the health for healthier shift work including sleep hygiene, physical activity, food intake, and control of light types and levels.

\section{6b NIGHT SHIFT-WORK AND BREAST CANCER RISK - WHAT IS THE EVIDENCE?}

Johnni Hansen*. Danish Cancer Society Research Centre, Copenhagen Denmark

\subsection{6/oemed-2018-ICOHabstracts. 1376}

Introduction Night work is increasingly common in the modern world. In 2007, the International Agency for Research on Cancer (IARC) classified shift work that involves circadian disruption as probably carcinogenic to humans based on limited evidence from eight epidemiologic studies on breast cancer, in addition to sufficient evidence from animal experiments. The overall evidence was further supported by increased risk in studies of flight attendants, who may be exposed to both shift-work and jet-lag, though night work was not the primary focus in these studies.

Methods Based on a critical review of the scientific literature it is the aim of to present the current epidemiologic evidence and to identify eventual threshold effects, e.g. by duration of night shifts, number of consecutive shifts and menopausal status.

Results After 2007, over 18 new studies with focus on night work and breast cancer are published, which more than triples the number of studies focusing on this issue since the IARC evaluation. The assessment of night shift work is different in all of the studies, which may attenuate the observed increased risk and hinders meta-analysis. There is some evidence that long duration and or high number of consecutive night shifts has impact on the magnitude of increased breast cancer risk. There is also some evidence that increased risk is primarily observed in women who have had nigh work in young adulthood, and in pre-menopausal women.

Conclusion Overall, there is a tendency of increased risk of breast cancer after long-term night shift or after shorter periods with many consecutive shifts. More studies using harmonised definitions of night work metrics is needed. Finally, evidence based preventive interventions are warranted.

\section{C NIGHTSHIFT WORK AND PROSTATE CANCER AMONG HONG KONG CHINESE MEN}

${ }^{1}$ Lap Ah Tse ${ }^{*},{ }^{1}$ Feng Wang, ${ }^{1}$ Priscilla Ming Yi Lee, ${ }^{2}$ Wing Ming Ho, ${ }^{3}$ Chi Fai Ng. ${ }^{1}$ Division of Occupational and Environmental Health, JC School of Public Health and Primary Care, The Chinese University of Hong Kong, Hong Kong SAR, China; ${ }^{2}$ Department of Clinical Oncology, Prince of Wales Hospital, Hong Kong SAR, China; ${ }^{3}$ SH Ho Urology Centre, Department of Surgery, The Chinese University of Hong Kong, Hong Kong SAR, China

\subsection{6/oemed-2018-ICOHabstracts. 1377}

Introduction A positive association between nightshift work and prostate cancer risk has been reported in epidemiological studies, but the findings have been mixed. Also, none of the previous studies has attempted to sufficiently consider the possible confounding effect from dietary sources including 